\title{
Moments of the stationary distribution of subcritical multitype Galton-Watson processes with immigration
}

\author{
Péter Kevei* $^{*}$ and Péter Wiandt ${ }^{\dagger}$
}

\begin{abstract}
In this short note we obtain necessary and sufficient conditions for the existence of the moments of the stationary distribution of a subcritical multitype Galton-Watson process with immigration.

Keywords: branching process with immigration, stationary distribution, moments

MSC2010: 60J80, 60G10
\end{abstract}

\section{Introduction}

Let $\left(\mathbf{X}_{n}\right)_{n \geq 0}=\left(X_{n, 1}, \ldots, X_{n, d}\right)_{n \geq 0}$ be a $d$-type Galton-Watson process with immigration (GWI), defined as

$$
\begin{aligned}
& \mathbf{X}_{n}=\sum_{j=1}^{d} \sum_{i=1}^{X_{n-1, j}} \mathbf{A}_{n, i ; j}+\mathbf{B}_{n}, \quad n=1, \ldots, \\
& \mathbf{X}_{0}=\mathbf{0}=(0, \ldots, 0),
\end{aligned}
$$

where $\mathbf{A}_{n, i ; j}, \mathbf{B}_{n}, n \geq 1, i \geq 1, j \in\{1, \ldots, d\}$, are independent $d$-dimensional random vectors with integer coordinates such that $\left\{\mathbf{A}_{n, i ; j}: n \geq 1, i \geq 1\right\}$ is an identically distributed sequence of random variables for each $j \in$ $\{1, \ldots, d\}$, and $\mathbf{B}_{n}, n \geq 1$, are identically distributed. Here $X_{n, j}$ is the number of $j$-type individuals in generation $n, \mathbf{A}_{n, i ; j}$ are the offsprings produced by the $i$ th individual of type $j$ in generation $n-1$, and $\mathbf{B}_{n}$ are the immigrants. In what follows, vectors, both deterministic and random, are denoted by boldface letters, and are meant as $d$-dimensional row vectors.

\footnotetext{
*Bolyai Institute, University of Szeged, Aradi vértanúk tere 1, 6720 Szeged, Hungary; e-mail: kevei@math.u-szeged.hu

${ }^{\dagger}$ Bolyai Institute, University of Szeged, Aradi vértanúk tere 1, 6720 Szeged, Hungary; e-mail: wpeti88@gmail.com
} 
Branching processes play an important role in models of genetics, molecular biology, physics and computer science. As a main reference on branching processes we refer to the classical books by Athreya and Ney [1, by Mode [7] and by Haccou et al. [5].

Multitype Galton-Watson processes with immigration was introduced and studied by Quine [8]. In [8] necessary and sufficient condition was given for the existence of stationary distribution in the subcritical case. A complete answer for the existence of a limiting stationary distribution for multitype GWI was obtained by Kaplan [6]. Also Mode [7] gave a sufficient condition for the existence of a stationary distribution. Multitype GWI processes in random environment were investigated by Roitershtein 9], Roitershtein and Zhong [10, Wang [12, to mention just a few.

However, interestingly enough, conditions for the existence of moments of the stationary distribution are not known except in special cases. Explicit formula for the variance was obtained by Quine [8] and for the third moment very recently by Barczy et al. [2, Lemma 1]. In the unpublished work by Szűcs [11] under ergodicity assumptions the existence of general moments of the stationary distribution were obtained for multitype GWI processes. Even in the single type case, we are only aware of the recent results by Buraczewski and Dyszewski [4] on Galton-Watson processes in random environment (without immigration), from which the existence of certain moments can be deduced, see Lemma 3.1 in [4], and by Basrak and Kevei [3, Lemma 1] on GWI processes in random environment.

The aim of the present paper is to fill this gap and obtain necessary and sufficient conditions for the existence of the moments of the stationary distribution.

\section{Results}

We assume that the offspring means are finite, and let $M$ denote the offspring mean matrix

$$
M=\left(\begin{array}{c}
\mathbb{E} \mathbf{A}_{1,1 ; 1} \\
\vdots \\
\mathbb{E} \mathbf{A}_{1,1 ; d}
\end{array}\right)=\left(\begin{array}{ccc}
m_{1,1} & \ldots & m_{1, d} \\
\vdots & \ddots & \vdots \\
m_{d, 1} & \ldots & m_{d, d}
\end{array}\right)
$$

that is $m_{i, j}$ is the mean offsprings of type $j$ produced by an individual of type $i$. Let $\rho$ denote the spectral radius of $M$, and assume that the process is subcritical, i.e. $\rho<1$. Without immigration a subcritical process dies out almost surely exponentially fast. While, Quine [8] showed that immigration with finite logarithmic moments ensures the existence of a stationary 
distribution. To ease notation we introduce the random operators $\theta_{n}$ as

$$
\theta_{n} \circ \mathbf{k}=\sum_{j=1}^{d} \sum_{i=1}^{k_{j}} \mathbf{A}_{n, i ; j}, \quad \mathbf{k}=\left(k_{1}, \ldots, k_{d}\right) .
$$

We slightly abuse the notation writing $\theta_{n} \circ\left(\mathbf{k}_{1}+\mathbf{k}_{2}\right)=\theta_{n} \circ \mathbf{k}_{1}+\theta_{n} \circ \mathbf{k}_{2}$, where on the right-hand side the two summands are independent. Further, write $\Pi_{n}=\theta_{1} \circ \ldots \circ \theta_{n}$, for $n \geq 1$, and $\Pi_{0}=\mathrm{Id}$. With this notation (1) can be written as $\mathbf{X}_{n}=\theta_{n} \circ \mathbf{X}_{n-1}+\mathbf{B}_{n}$. Iteration gives that the stationary distribution can be represented in distribution as

$$
\mathbf{Y}=\mathbf{B}_{1}+\theta_{1} \circ \mathbf{B}_{2}+\theta_{1} \circ \theta_{2} \circ \mathbf{B}_{3}+\ldots=\sum_{i=0}^{\infty} \Pi_{i} \circ \mathbf{B}_{i+1},
$$

provided that the infinite sum exists. This corresponds to formula (16) in [8] in terms of generating functions.

Introduce the notation $\|\mathbf{x}\|=\sum_{j=1}^{d}\left|x_{j}\right|$ for the $\ell^{1}$ norm of a vector in $\mathbb{R}^{d}$, and also for the generated matrix norm $\|A\|=\sup _{\|\mathbf{x}\|=1}|\mathbf{x} A|$. Note that $\|A\|$ is the maximum absolute row sum, since we multiply from the left.

Our main result is the following.

Theorem. Let $\left(\mathbf{X}_{n}\right)_{n \geq 0}$ be a d-type, subcritical Galton-Watson process with immigration. If $\mathbb{E}\left\|\mathbf{A}_{1,1 ; i}\right\|^{\max \{\alpha, 1\}}<\infty$ for all $i \in\{1, \ldots, d\}$, and $\mathbb{E}\|\mathbf{B}\|^{\alpha}<$ $\infty$ for some $\alpha>0$, then $\mathbb{E}\|\mathbf{Y}\|^{\alpha}<\infty$. In particular, each component of $\mathbf{Y}$ has finite moment of order $\alpha$.

Note that by the subcriticality assumption $\mathbb{E}\left\|\mathbf{A}_{1,1 ; i}\right\|<\infty$ for any $i \in$ $\{1, \ldots, d\}$. Thus, even for $\alpha<1$ we assume the existence of the offspring mean. However, the immigration distribution might have infinite mean.

Also note that we do not assume the existence of a unique stationary distribution. We only use that the conditions of the theorem implies that the infinite sum in (4) is a.s. finite.

Finally, we mention that these are only sufficient conditions. Clearly, if a type-1 particle never immigrates, then the distribution of $\mathbf{A}_{1,1 ; 1}$ does not matter in the stationary distribution. Otherwise, from formula (4) we see that our conditions are also necessary.

\section{Proof}

\subsection{The case $\alpha \geq 1$}

Let $\alpha \geq 1$ be fixed. First we prove the theorem under the additional assumption that

$$
\|M\|<1
$$


that is the row sums are less than 1 . Let $\mu_{j}=\sum_{\ell=1}^{d} m_{j, \ell}, j \in\{1, \ldots, d\}$.

We prove that

$$
M_{\alpha}(k)=\mathbb{E}\left\|\Pi_{k} \circ \mathbf{B}_{k+1}\right\|^{\alpha}
$$

decreases exponentially.

Recall (3). To ease notation we suppress the lower index. We have

$$
\|\theta \circ \mathbf{k}\|=\sum_{j=1}^{d} \sum_{i=1}^{k_{j}}\left\|\mathbf{A}_{i ; j}\right\|=\sum_{j=1}^{d} \sum_{i=1}^{k_{j}} \sum_{\ell=1}^{d} A_{i ; j}^{(\ell)}=: \sum_{j=1}^{d} S_{k_{j} ; j},
$$

where $\left\|\mathbf{A}_{i ; j}\right\|=\sum_{\ell=1}^{d} A_{i ; j}^{(\ell)}$ is the number of all the offsprings of the $i$ th individual of type $j$, with $A_{i ; j}^{(\ell)}$ being the number of $\ell$-type offsprings, and $S_{k ; j}=\sum_{i=1}^{k}\left\|\mathbf{A}_{i ; j}\right\|$ is the sum of $k$ iid scalar random variables, with mean $\mathbb{E}\left\|\mathbf{A}_{1 ; j}\right\|=\sum_{\ell=1}^{d} m_{j, \ell}=\mu_{j}<1$.

For any $\bar{\mu} \in\left(\max _{1 \leq j \leq d} \mu_{j}, 1\right)$ there exists $k_{0}^{\prime}$ such that

$$
\mathbb{E}\left(\frac{S_{k ; j}}{k}\right)^{\alpha}<\bar{\mu} \text { for all } k \geq k_{0}^{\prime}, j \in\{1, \ldots, d\} .
$$

This follows from the strong law of large numbers combined with the uniform integrability of $S_{k ; j} / k$. Put

$$
\max _{1 \leq j \leq d} \max _{k<k_{0}^{\prime}} \mathbb{E}\left(\frac{S_{k ; j}}{k}\right)^{\alpha}=c_{0} .
$$

Since the function $x^{\alpha}$ is convex, we have

$$
\begin{aligned}
\left(\frac{\sum_{j=1}^{d} S_{k_{j} ; j}}{k_{1}+\ldots+k_{d}}\right)^{\alpha} & =\left(\sum_{j=1}^{d} \frac{k_{j}}{k_{1}+\ldots+k_{d}} \frac{S_{k_{j} ; j}}{k_{j}}\right)^{\alpha} \\
& \leq \sum_{j=1}^{d} \frac{k_{j}}{k_{1}+\ldots+k_{d}}\left(\frac{S_{k_{j} ; j}}{k_{j}}\right)^{\alpha} .
\end{aligned}
$$

Combined with (7) and (8) this implies

$$
\begin{aligned}
\mathbb{E}\|\theta \circ \mathbf{k}\|^{\alpha} & =\|\mathbf{k}\|^{\alpha} \mathbb{E}\left(\frac{\|\theta \circ \mathbf{k}\|}{\|\mathbf{k}\|}\right)^{\alpha} \\
& \leq\|\mathbf{k}\|^{\alpha} \sum_{j=1}^{d} \frac{k_{j}}{\|\mathbf{k}\|} \mathbb{E}\left(\frac{S_{k_{j} ; j}}{k_{j}}\right)^{\alpha} \\
& \leq\|\mathbf{k}\|^{\alpha} \sum_{j=1}^{d} \frac{k_{j}}{\|\mathbf{k}\|}\left[I\left(k_{j} \geq k_{0}^{\prime}\right) \bar{\mu}+I\left(k_{j}<k_{0}^{\prime}\right) c_{0}\right] \\
& \leq\|\mathbf{k}\|^{\alpha}\left(\bar{\mu}+\frac{k_{0}^{\prime} c_{0} d}{\|\mathbf{k}\|}\right),
\end{aligned}
$$


where $I(\cdot)$ stands for the indicator function. Choosing $k_{0}>2 k_{0}^{\prime} c_{0} d /(1-\bar{\mu})$ we obtain that

$$
\mathbb{E}\|\theta \circ \mathbf{k}\|^{\alpha} \leq \mu\|\mathbf{k}\|^{\alpha} \quad \text { whenever }\|\mathbf{k}\| \geq k_{0},
$$

with $\mu=(1+\bar{\mu}) / 2<1$.

Let $\mathbf{Z}$ be a random vector with nonnegative integer components, independent of the A's. Put

$$
c_{1}=\max _{|\mathbf{k}|<k_{0}} \mathbb{E}\|\theta \circ \mathbf{k}\|^{\alpha} .
$$

Then, by (9)

$$
\begin{aligned}
\mathbb{E}\|\theta \circ \mathbf{Z}\|^{\alpha} & =\sum_{\mathbf{k}} \mathbb{P}(\mathbf{Z}=\mathbf{k}) \mathbb{E}\|\theta \circ \mathbf{k}\|^{\alpha} \\
& =\sum_{\mathbf{k}:\|\mathbf{k}\| \geq k_{0}} \mathbb{P}(\mathbf{Z}=\mathbf{k}) \mathbb{E}\|\theta \circ \mathbf{k}\|^{\alpha}+\sum_{\mathbf{k}: 0<\|\mathbf{k}\|<k_{0}} \mathbb{P}(\mathbf{Z}=\mathbf{k}) \mathbb{E}\|\theta \circ \mathbf{k}\|^{\alpha} \\
& \leq \sum_{\mathbf{k}:\|\mathbf{k}\| \geq k_{0}} \mathbb{P}(\mathbf{Z}=\mathbf{k}) \mu\|\mathbf{k}\|^{\alpha}+\sum_{\mathbf{k}: 0<\|\mathbf{k}\|<k_{0}} \mathbb{P}(\mathbf{Z}=\mathbf{k}) c_{1} \\
& \leq \mu \mathbb{E}\|\mathbf{Z}\|^{\alpha}+c_{1} \mathbb{E}\|\mathbf{Z}\| .
\end{aligned}
$$

Turning back to $M_{\alpha}(k)$ in (6), we obtain the recursion

$$
M_{\alpha}(k) \leq \mu M_{\alpha}(k-1)+c_{1} M_{1}(k-1),
$$

with $\mu<1$. Note that $M_{1}(k)$ is the expectation of the total number of individuals in generation $k$ in a multitype Galton-Watson process without immigration, starting with $\mathbf{B}_{k+1}$ at generation 0. Therefore $M_{1}(k)=\left\|M^{k} \mathbb{E} \mathbf{B}\right\|$, which decreases exponentially fast. Thus, recursion (10) implies that for some $\nu \in(\mu, 1)$ and $C>0$

$$
M_{\alpha}(k) \leq C \nu^{k} \quad \text { for all } k \geq 0 .
$$

The statement now follows from Minkowski's inequality, as

$$
\begin{aligned}
\left(\mathbb{E}\|\mathbf{Y}\|^{\alpha}\right)^{1 / \alpha} & =\left(\mathbb{E}\left\|\sum_{k=0}^{\infty} \Pi_{k} \circ B_{k+1}\right\|^{\alpha}\right)^{1 / \alpha} \\
& \leq\left(\sum_{k=0}^{\infty} M_{\alpha}(k)\right)^{1 / \alpha}<\infty .
\end{aligned}
$$

The additional assumption in (5) can be omitted easily. By Gelfand's formula for the spectral radius we have

$$
\lim _{k \rightarrow \infty}\left\|M^{k}\right\|^{1 / k}=\rho,
$$


which is strictly less than 1 , by subcriticality. Thus, there exists $r$ such that $\left\|M^{r}\right\|<1$. The matrix $M^{r}$ is the mean matrix of the offspring distribution corresponding to $\Pi_{r}$, i.e. when we sample the process only in every $r$ th generation. Therefore, the previous argument gives that $M_{\alpha}(r k+i)$ in (2) decreases exponentially for each $i \in\{0,1, \ldots, r-1\}$. Clearly, then $M_{\alpha}(k)$ also decreases exponentially, and the result follows from Minkowski's inequality as above.

\subsection{The case $\alpha<1$}

This case is in fact simpler, but needs to be treated differently.

First, assume again that (5) holds. We use the same notations as above. Now $x^{\alpha}$ is concave, thus by Jensen's inequality for any $\mathbf{k}$

$$
\begin{aligned}
\mathbb{E}\|\theta \circ \mathbf{k}\|^{\alpha} & =\mathbb{E}\left(\sum_{j=1}^{d} S_{k_{j} ; j}\right)^{\alpha} \leq\left(\mathbb{E} \sum_{j=1}^{d} S_{k_{j} ; j}\right)^{\alpha} \\
& =\left(\sum_{j=1}^{d} k_{j} \mu_{j}\right)^{\alpha} \leq \mu\|\mathbf{k}\|^{\alpha},
\end{aligned}
$$

with $\mu=\max _{1 \leq j \leq d} \mu_{j}$, implying

$$
\begin{aligned}
\mathbb{E}\|\theta \circ \mathbf{Z}\| & =\sum_{\mathbf{k}} \mathbb{P}(\mathbf{Z}=\mathbf{k}) \mathbb{E}\|\theta \circ \mathbf{k}\|^{\alpha} \\
& \leq \sum_{\mathbf{k}} \mathbb{P}(\mathbf{Z}=\mathbf{k}) \mu\|\mathbf{k}\|^{\alpha} \leq \mu \mathbb{E}\|\mathbf{Z}\|^{\alpha} .
\end{aligned}
$$

Therefore, the exponential decrease of $M_{\alpha}(k)$ in (6) follows. By subadditivity we have

$$
\mathbb{E}\|\mathbf{Y}\|^{\alpha}=\mathbb{E}\left\|\sum_{k=0}^{\infty} \Pi_{k} \circ B_{k+1}\right\|^{\alpha} \leq \sum_{k=0}^{\infty} M_{\alpha}(k)<\infty,
$$

as claimed.

Condition (5) can be omitted the same way as before.

Acknowledgement. Péter Kevei is supported by the János Bolyai Research Scholarship of the Hungarian Academy of Sciences, by the NKFIH grant FK124141, and by the EU-funded Hungarian grant EFOP-3.6.1-162016-00008. Péter Wiandt is supported by the Ministry of Innovation and Technology grant UNKP-19-2-SZTE-139, by the Ministry of Human Capacities, Hungary grant 20391-3/2018/FEKUSTRAT, and by the EU-funded Hungarian grant EFOP-3.6.2-16-2017-00015. 


\section{References}

[1] K. B. Athreya and P. E. Ney. Branching processes. Springer-Verlag, New York-Heidelberg, 1972. Die Grundlehren der mathematischen Wissenschaften, Band 196.

[2] M. Barczy, F. K. Nedényi, and G. Pap. On aggregation of multitype Galton-Watson branching processes with immigration. Mod. Stoch. Theory Appl., 5(1):53-79, 2018.

[3] B. Basrak and P. Kevei. Limit theorems for branching processes with immigration in a random environment. https://arxiv.org/abs/2002.0063, 2020.

[4] D. Buraczewski and P. Dyszewski. Precise large deviation estimates for branching process in random environment. https://arxiv.org/abs/1706.03874v1, 2019.

[5] P. Haccou, P. Jagers, and V. A. Vatutin. Branching processes: variation, growth, and extinction of populations, volume 5 of Cambridge Studies in Adaptive Dynamics. Cambridge University Press, Cambridge; IIASA, Laxenburg, 2007.

[6] N. Kaplan. The multitype Galton-Watson process with immigration. Ann. Probability, 1(6):947-953, 1973.

[7] C. J. Mode. Multitype branching processes. Theory and applications. Modern Analytic and Computational Methods in Science and Mathematics, No. 34. American Elsevier Publishing Co., Inc., New York, 1971.

[8] M. P. Quine. The multi-type Galton-Watson process with immigration. J. Appl. Probability, 7:411-422, 1970.

[9] A. Roitershtein. A note on multitype branching processes with immigration in a random environment. Ann. Probab., 35(4):1573-1592, 2007.

[10] A. Roitershtein and Z. Zhong. On random coefficient INAR(1) processes. Sci. China Math., 56(1):177-200, 2013.

[11] G. Szűcs. Ergodic properties of subcritical multitype Galton-Watson processes. Available on arXiv: https://arxiv.org/abs/1402.5539.

[12] H. M. Wang. A note on multitype branching process with bounded immigration in random environment. Acta Math. Sin. (Engl. Ser.), 29(6):1095-1110, 2013. 